\title{
Sustainable multi-objective scheduling for automatic guided vehicle and flexible manufacturing system by a grey wolf optimization algorithm
}

\author{
V. K. Chawla ${ }^{a *}$, Arindam Kumar Chanda ${ }^{\mathrm{b}}$ and Surjit Angra ${ }^{\mathrm{c}}$
}

${ }^{a}$ Indira Gandhi Delhi Technical University for Women, India

${ }^{b}$ G.B. Pant Engineering College, India

${ }^{c}$ NIT Kurukshetra, India

\section{H R O N I C L E}

\section{Article history:}

Received: January 1, 2018

Received in revised format: June

16,2018

Accepted: June 20, 2018

Available online:

June 20, 2018

Keywords:

Automatic guided vehicles

Flexible manufacturing system

Grey wolf optimization

Sustainable multi-objective scheduling \begin{abstract}
A B S T R A C T
The simultaneous scheduling decisions between production systems and material handling systems are highly significant for a substantial reduction in makespan and improvement in throughput of flexible manufacturing system resources. In the absence of appropriate scheduling of production resources, the optimum utilization of FMS resources is not harnessed which turns into wastage of resources. In the present study, investigations are carried out for the sustainable multiobjective scheduling of automatic guided vehicle and flexible manufacturing system by the application of a grey wolf optimization algorithm (GWO). Initially the Giffler and Thompson (GT) algorithm [Giffler, B., \& Thompson, G. L. (1960). Algorithms for solving production-scheduling problems. Operations research, 8(4), 487-503.] along with four different priority hybrid dispatching rules (PHDRs) are applied for the development of the production center schedule thereafter the grey wolf optimization algorithm is applied for the yield of the sustainable multi-objective scheduling of automatic guided vehicles (AGVs) and the FMS together with an objective to minimize the total distance travel and number of backtracking of cruising automatic guided vehicle in the $U$ type flexible manufacturing system facility. The applied methodology is evaluated by conducting computational experiments on a benchmark flexible manufacturing system configuration considered from the literature. The results obtained from the computational experiments clearly show that the proposed application of grey wolf optimization algorithm outperforms the other applied procedures in the literature.
\end{abstract}

\section{Introduction}

In the global competition, the sustainable and profitable manufacturing operations are one of the biggest challenges of the present times to deal with. Moreover, the customized demands of customers for different products with high quality at a reasonable price can only be fulfilled with a high degree of flexibility and quick change-overs in the manufacturing operations. The flexible manufacturing systems (FMS) appeared as a right alternative to cater all customized production demands of the customers in a justified

* Corresponding author.

E-mail address: kumar6150046@nitkkr.ac.in (V. K. Chawla) 
manner. In order to deliver the right quantity and quality of products in line with the product specifications, it becomes highly essential to maintain zero waiting time of FMS resources i.e. the FMS resources should work continuously with no waiting time and idle time of the production centers etc. One way to achieve this objective is to develop and apply sustainable simultaneous schedules to the FMS resources (Prakash et al., 2011; Jahromi et al., 2017; Chawla et al., 2018a; 2018b; Angra et al., 2018). The FMS constitutes a set of advanced programmable production centers which are controlled by the computers. The production centers in the FMS are highly flexible and are capable to carry out versatile production operations on a part within the tight tolerances. The capability of achieving precision, accuracy, and versatility in the production operations with high flexibility makes the application of FMS a popular choice among the competitive manufacturing organizations. The FMS facility generally comprises of robots, horizontal, vertical and universal machining centers etc. Apart from different types of production centers the FMS also consist of automatic guided vehicle systems (AGVs) to carry out the material transfer operations from one production center to other or to the buffer area in the FMS configuration (Egbelu \& Tanchoco, 1986; Gaskins \& Tanchoco, 1987; Sen et al., 2017). The sustainable simultaneous scheduling of AGVs and production centers in the FMS can increase the throughput and reduce makespan of FMS which can further assure the sustainable profits from the FMS operations. The sustainable simultaneous scheduling decisions for real-time manufacturing systems are based on dynamic manufacturing conditions (Taghaboni \& Tanchoco, 1995; Rajotia et al., 1998). The sustainable multiobjective simultaneous scheduling can be advantageously applied between AGVs and FMS for high operational control on the production of jobs in the FMS (Tiwari \& Harding, 2011; Udhayakumar \& Kumanan, 2012). In light of benefits of sustainable multi-objective simultaneous scheduling of FMS resources, in this study, an attempt is made to investigate the output of simultaneous production scheduling and AGVs scheduling for the reported FMS configuration. The production centers scheduling is carried out with the Giffler and Thompson algorithm under four types of priority hybrid dispatching rules then the developed schedule is confirmed with AGV schedule and optimized by the application of grey wolf optimization algorithm to simultaneously minimize the gross AGV distance travel and number of backtracking in the FMS configuration.

This paper comprises of five more sections, section 2 presents the literature review and identifies the research gap, section 3 describes the problem model and assumptions. The grey wolf optimization algorithm is discussed in section 4 . Section 5 presents the proposed procedure on the numerical example from the literature and output results. Finally, the paper is concluded in section 6 along with possible future research directions.

\section{Literature Review}

The AGVs are commonly deployed for material handling activities in the FMS facility. The AGVs carries and transfer raw, semi-finished and finished jobs while moving on the predefined layout at various locations of the FMS facility according to the requirement of FMS resources and AGV schedule. An enumerative method for scheduling of " $n$ " jobs on " $m$ " machines was introduced by Giffler and Thompson (1960). Sabuncuoglu and Hommertzheim (1992) proposed a dispatching algorithm to select jobs for the idle work centers and AGVs simultaneously. Authors performed series of simulation experiment and considered mean flow time, mean tardiness as governing factors for comparing the performance of various job selection dispatching rules. Suleyman and Ihsan (1993) proposed simultaneous scheduling procedure for work centers and AGVs in FMS facility by application of beam search algorithm. Lee \& Cesare (1994) considered a job shop facility for investigating integrated offline scheduling of machine centers and AGVs. Ulusoy and Bilge (1993) described significance and advantages of the simultaneous scheduling of MHS and FMS and proposed a procedure to integrate the FMS overall production schedule with the AGVs schedule. Kim and Hwang (1999) considered FMS throughput as main criteria to investigate performance and effectiveness of the proposed dispatching algorithm by conducting simulation experiment. Sadjadi and Makui (2002) developed a novel algorithm to solve static and dynamic complexity in the production planning problems. Haq et al. (2003) attempted to solve multilevel simultaneous 
scheduling problem of AGVs and FMS by application of simulated annealing and genetic algorithm. A hybrid genetic algorithm for simultaneous scheduling of job shop and AGVs with an objective to lower down makespan of entire FMS was proposed by Abdelmaguid et al. (2004) it was observed that the proposed hybrid genetic algorithm was made up of a combination of genetic algorithm and vehicle assignment algorithm. Jerald et al. (2005) proposed an adaptive genetic algorithm (AGA) for simultaneous scheduling of work parts and AGVs in the FMS. Ho and Liu (2009) described three main issues in AGVs scheduling and dispatching namely i) delivery-dispatching issue ii) load selection issue and iii) taskdetermination issue. Authors conducted simulation experiment for simultaneous task-determination and delivery dispatching issues in the FMS. Xia \& Wu (2006) minimized makespan of FMS by the application of hybrid particle swarm optimization algorithm for scheduling of production systems. Huang \& Liao (2008) explored possibilities to apply ant colony optimization (ACO) algorithm and tabu search algorithm for optimum scheduling of job shop production system further authors found that the proposed procedure outperforms conventional scheduling procedures. An artificial intelligence system for multiobjective scheduling of automatic storage and retrieval system, Jobs and AGVs was investigated by Gnanavelbabu et al. (2008). Kashan and Karimi (2009) proposed PSO algorithm for minimizing makespan of FMS and optimum job assignment to the work center of the FMS. Sadrabadi and Sadjadi (2009) investigated an interactive algorithm for the solution of the multi-objective problems. Moghaddam et al. (2012) considered uncertain customer requirements and unknown distributions to solve the vehicle routing problem. An advanced particle swarm optimization algorithm was applied by the authors for the solution of the uncertain vehicle routing problem. In order to evaluate different flexibilities of FMS Jain and Raj (2013) integrated simple additive weighting (SAW) and weighted product method (WPM) with analytical hierarchy process (AHP). Singh \& Khan (2016) proposed an analytical model for the solution of loading and unloading problem in the FMS with minimum computational time. Wang et al. (2016) analyzed random arrival of work parts by a discrete even simulation study.In their simulation study authors applied apart launching dispatching rule-based tardiness estimation method. From the simulation results, it was revealed that proposed tardiness estimation method outperforms other dispatching rules. Chawla et al. (2018c, 2018d) applied modified memetic particle swarm optimization algorithm and a grey wolf optimization algorithm (Mirjalili et al., 2014, 2016) to yield an initial feasible solution for scheduling of multi-load AGVs and also to optimize the AGV fleet size for the FMS respectively.

The literature review identifies a potential research gap for the development of sustainable simultaneous multi-objective production schedule confirming to the AGV schedule under the priority hybrid dispatching rules optimized by grey wolf optimization algorithm. Here in the present study, an attempt is made to bridge the aforesaid research gap by carrying out the sustainable simultaneous multi-objective scheduling between the production centers and AGVs under priority hybrid dispatching rules so as to minimize the back-tracking and distance travel of AGVs by application of the grey wolf optimization algorithm for the U type FMS configuration.

\section{Problem Definition}

\subsection{Assumptions}

Following assumptions are considered.

1. The job entry and exit is only through the load-unload station.

2. More than one processing sequence is possible for a job.

3. The pick and drop time is deterministic.

4. In case of no pending pick and drop request, the AGV will wait at its location until a new request is received.

5. The FMS configuration, number of production centers and buffer details are known.

6. Setup time on production center for all the operations is assumed to be zero. 
The different kinds of " $E$ " parts are to be produced on " $K$ " types of production centers installed in the FMS configuration as portrayed in figure 3 . In order to allocate parts to the production centers, the partproduction center incidence matrix is referred. The part-production center matrix also informs about the production steps for a part $E_{i}$ that to be performed on production center $K_{i}$. The part routing in the FMS configuration is developed by using four kinds of priority hybrid dispatching rules (PHDRs) and the Giffler and Thompson (GT) algorithm. The PHDR is a yield from a productive blend of either two static dispatching rules or two dynamic dispatching rules so as to minimize the overall performance criteria. The parameter of single priority dispatching rule (static or dynamic) is multiplied for the development of a new priority hybrid dispatching rule Kaban et al. (2012). For instance, to develop a priority hybrid dispatching rule, SPS and SPT, (shortest processing sequence, SPS - static dispatch rule and shortest processing time, SPT - dynamic dispatch rule) the value of " $V$ " are calculated as:

$V=P T_{i j} \times P S_{i}$ (Kaban et al., 2012)

where,

$i \quad$ Set of production operations;

$j \quad$ Set of production centers;

$P T$ The value of time for production operation $i$ on the production center $j$;

PS The value of total sequence required in the production operation $i$ for the completion of the production operation.

The PHDR with a minimum value of " $V$ " is applied at the top priority. In order to yield a sustainable simultaneous multi-objective schedule, the routing flexibility of parts under production is considered from the application of PHDRs with GT algorithm and after evaluation of the part routings, the best part route is considered for an input to the automatic guided vehicle system so as to simultaneously reduce the distance travel and back-tracking of the AGV. In order to develop a sustainable simultaneous schedule, the two objectives i.e. minimizing the gross travel distance and back-tracking of AGVs are optimized simultaneously by applying the grey wolf optimization algorithm (GWO). To solve the aforesaid multi-objective problem, the problem is transformed into a single objective problem by the weighted sum approach also mentioned in the equation.

$\min Z=p_{1} D+p_{2} B$

where,

$D=\sum_{i=1}^{E} \sum_{j=1}^{K} \sum_{k=1}^{K} d_{i j k} ; d_{i j k}=0 ;$ if $j=k ;$

$B=\sum_{i=1}^{E} \sum_{j=1}^{K} \sum_{k=1}^{K} b_{i j k} ; b_{i j k}=\left\{\begin{array}{l}1 ; \text { if } j>k \\ 0 ; \text { otherwise }\end{array}\right\}$

$p_{1}=0.6$

$p_{2}=0.4$

where,

$d_{i j k} \quad$ The distance travel of AGV in part transfer;

$b_{i j k} \quad$ The back-tracking by AGV in the part transfer;

$D \quad$ Aggregate distance travel of AGV;

$B$ Aggregate back-tracking;

$Z$ The objective function;

$p_{1} \quad$ The weight applied to the distance travel, Deb (2001);

$p_{2} \quad$ The weight applied for the back-tracking, Deb (2001);

The following priority hybrid dispatching rules are applied as an input to the Giffler and Thomson algorithm for the development of production center schedule. 
1) SPS and ECT (Shortest Processing Sequence + Earliest Creation Time)

In SPS+ECT hybrid dispatching rule the SPS is the static rule and ECT is dynamic dispatching rule.

2) SWT + SPS (Shortest Waiting Time + Shortest Process Sequence)

In SWT + SPS hybrid dispatching rule the SWT is the dynamic rule and SPS is static dispatching rule.

3) LWT + LPS (Longest Waiting Time + Longest Process Sequence)

In LWT+LPS hybrid dispatching rule the LWT is a dynamic dispatching rule and LPS is a static dispatching rule.

4) SPT + SPS (Shortest Processing Time + Shortest Process Sequence)

In SPT + SPS hybrid dispatching rule, the SPT is a static dispatching rule and SPS is a static dispatching rule.

\section{Grey Wolf Optimization Algorithm}

The grey wolf optimization (GWO) algorithm works on the leadership hierarchy and the intelligent hunting process exhibited by the grey wolves. In the GWO algorithm, four kinds of grey wolves namely alpha, beta, delta, and omega are considered for the simulation of the social hierarchy. The main steps comprising in the algorithm are a search of prey, encircle the prey, attack the prey and then hunt the prey (Mirzalili et al., 2014 and Mirzalili et al., 2016). The four kinds of grey wolves also represent the four fitness functions also namely: alpha, beta, gamma, and omega. During the iteration of the algorithm, the survival of the fittest solution is considered as alpha $(\alpha)$ thereafter, the second and the third best solutions are considered to be beta $(\beta)$ and delta $(\Delta)$, respectively. The rest of the solution is considered to be omega $(\omega)$ (Mirzalili et al., 2014; Mirzalili et al., 2016 and Bozorg-Haddad, 2017). The encircling behavior of grey wolf algorithm is modeled in the following equation:

$$
\begin{aligned}
& \vec{D}=|\vec{C} \cdot \vec{X} p(t)-\vec{X}(t)| \\
& \vec{X}(t+1)=\vec{X} p(t)-\vec{A} \cdot \vec{D}
\end{aligned}
$$

where,

$t=$ iteration number,

$\vec{A}$ and $\vec{C}=$ coefficient vectors of the bootstrap program,

$\vec{X} p(t)=$ prey positioning vector.

$\vec{X}=$ grey wolf's positioning vector.

$\vec{D}=$ Calculated vector, to specify the new position of the grey wolf.

$\vec{A}=2 \vec{a} \cdot \vec{r}_{1}-\vec{a}$

$\vec{C}=2 \vec{r}_{2}$

$\vec{a}=$ The linear decrease of vector set from 2 to 0 during the iteration.

$\vec{r}_{1}$ and $\vec{r}_{2}=$ The random vectors in $[0,1]$.

A grey wolf at $(x, y)$ position can move and update its position according to the prey position $\left(x^{\prime}, y^{\prime}\right)$. The positioning of the best agent can be carried out with respect to the present positioning of grey and prey by controlling $\vec{A}$ and $\vec{C}$. The hunting mechanism of grey wolves is simulated by considering that the wolves will keep on attacking the prey till the prey stops its movement. The hunting behavior of grey wolves is modeled and simulated considering that the value of $\vec{a}$ decrease during the iterations and the fluctuation rate of $\vec{A}$ also decrease with progress in the iterations. In the algorithm, the alpha ( $\alpha$, best agent) beta $(\beta)$ and delta $(\Delta)$ keeps more information about the position of the prey. Hence the GWO algorithm stores the three best solutions and after that, it initiates omega $(\omega)$, the fourth agent to update its position so as to achieve the best position in the search space further optimizing the yield of results. The alpha $(\alpha)$, beta $(\beta)$ and delta $(\Delta)$ estimate the prey's location and other wolves such as omega $(\omega)$ 
update their position randomly around the prey. The process of grey wolf optimizing algorithm is portrayed in figure las flowchart for grey wolf optimization algorithm and the pseudo-code of GWO is shown in Fig. 2. The GWO algorithm's hunting mechanism is modeled in following equations.

$$
\begin{aligned}
& \vec{D}_{\alpha}=\left|\vec{C}_{1} \cdot \vec{X}_{\alpha}-\vec{X}\right| \\
& \vec{D}_{\beta}=\left|\vec{C}_{2} \cdot \vec{X}_{\beta}-\vec{X}\right| \\
& \vec{D}_{\Delta}=\left|\vec{C}_{3} \cdot \vec{X}_{\Delta}-\vec{X}\right| \\
& \vec{X}_{1}=\vec{X}_{\alpha}-A_{1} .\left(\vec{D}_{\alpha}\right) \\
& \vec{X}_{2}=\vec{X}_{\beta}-A_{2} \cdot\left(\vec{D}_{\beta}\right) \\
& \vec{X}_{3}=\vec{X}_{\Delta}-A_{3} \cdot\left(\vec{D}_{\Delta}\right) \\
& \vec{X}(t+1)=\frac{\vec{X}_{1}+\vec{X}_{2}+\vec{X}_{3}}{3}
\end{aligned}
$$

The GWO algorithm has several benefits over the other metaheuristic techniques.

1. Implementation is easy due to the simple structure.

2. Less storage required than other technique.

3. Convergence of algorithm is fast due to the continuous reduction of search space and the reduction of decision variables.

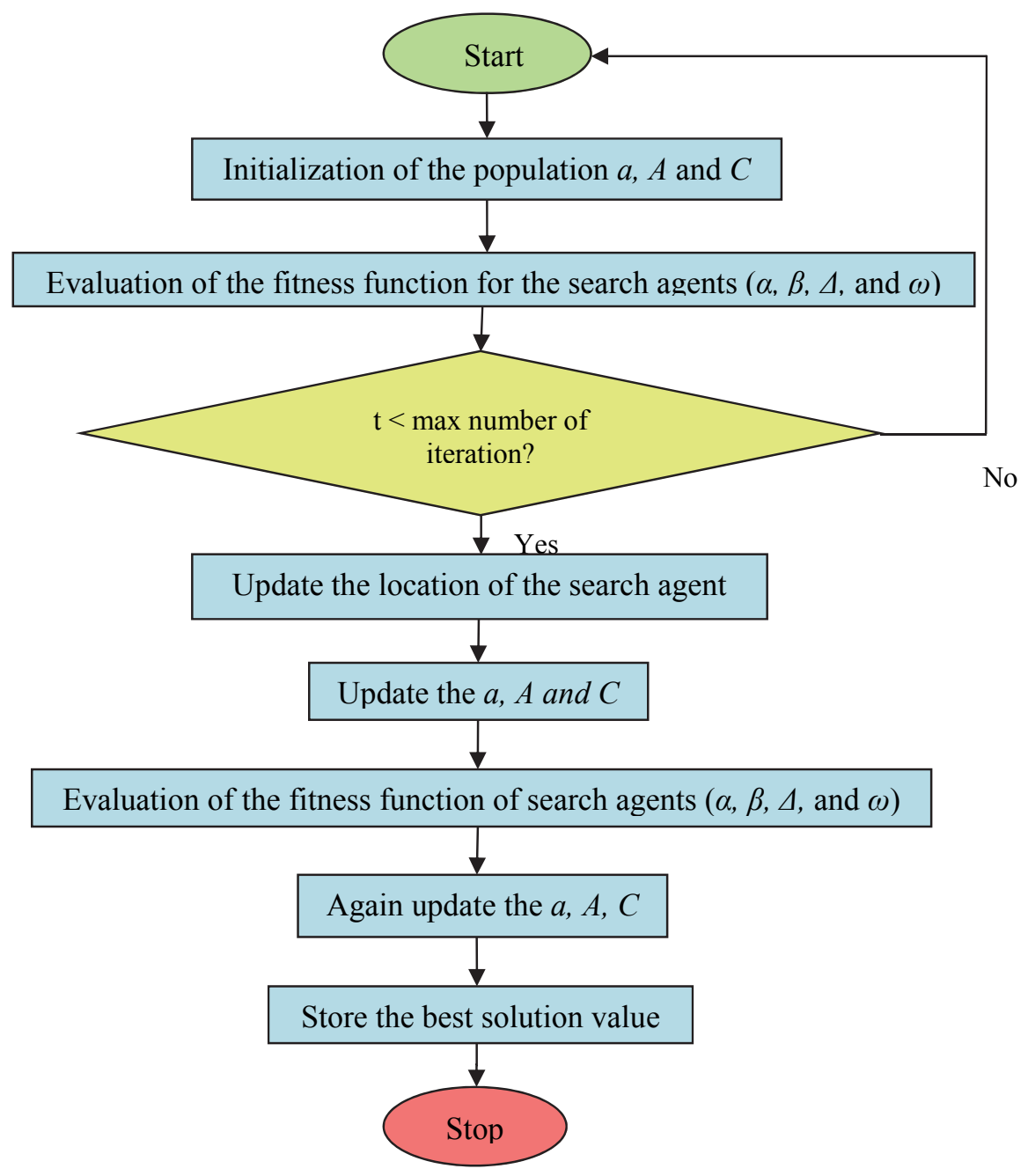

Fig. 1. Flowchart of grey wolf optimization algorithm 


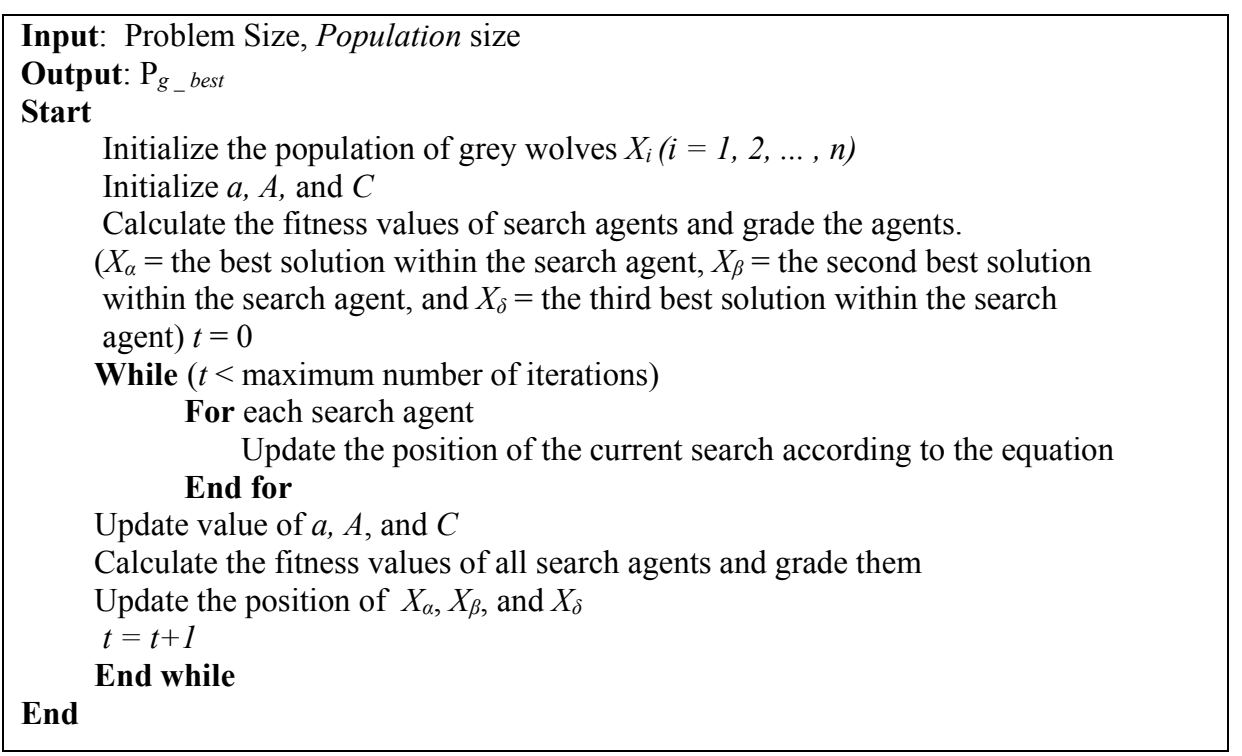

Fig. 2. Pseudo-code of grey wolf optimization algorithm

\section{Numerical Example}

The multi-objective simultaneous scheduling of U type FMS layout and AGVs is portrayed in figure 3 and also presented in Table 1. The FMS layout is referred from the literature Haq et al. (2003) as a benchmark problem.

Table 1

Description of the U-type FMS layout

\begin{tabular}{ccccc}
\hline Layout & No. of Production centers & No. of parts & Load-Unload Centre & No. of AGV \\
\hline U type & 9 & 2 & 2 & 1 \\
\hline
\end{tabular}

The nine production centers produce two types of parts in the FMS layout. The part operation sequence and their routing option are presented in Table 2 and production time on different production center are mentioned in Table 3. The production process for both of the parts in four possible combinations along with yield of makespan is also mentioned in Table 4.

Table 2

Part-machine centre incidence matrix

\begin{tabular}{ccccccccccc}
\hline \multirow{2}{*}{ Part types } & \multicolumn{1}{c}{ Operation sequence } \\
\cline { 2 - 10 } & 1 & 2 & 3 & 4 & 5 & 6 & 7 & 8 & K8 \\
\hline \multirow{2}{*}{ E1 } & K2 & K5 & K3 & K1 & K8 & K4 & K6 & K9 & K7 \\
& K2 & K6 & K3 & K5 & K1 & K4 & K9 & K7 & K8 \\
\multirow{2}{*}{ E2 } & K6 & K2 & K1 & K7 & K8 & K3 & K4 & K9 & K5 \\
& K6 & K9 & K5 & K3 & K1 & K4 & K7 & K8 & K2 \\
\hline
\end{tabular}

Table 3

Operation time of parts at the production center

\begin{tabular}{|c|c|c|c|c|c|c|c|c|c|}
\hline \multirow{2}{*}{ Part types } & \multicolumn{9}{|c|}{ Operation time } \\
\hline & K1 & $\mathrm{K} 2$ & K3 & $\mathrm{K} 4$ & K5 & K6 & K7 & K8 & K9 \\
\hline E1 & 62 & 9 & 89 & 87 & 10 & 66 & 95 & 49 & 82 \\
\hline E2 & 30 & 95 & 58 & 61 & 58 & 60 & 17 & 8 & 5 \\
\hline
\end{tabular}


The priority dispatching rules (PDRs) considered in literature Haq et al. (2003) are as follows:

1. SPT - Shortest processing time for the part.

2. LPT-Longest processing time for the part.

3. LOR-Least number of operations remaining for the part.

4. MOR - Most number of operations remaining for the part.

5. LWR - Least amount of work remaining on the part.

6. MWR-Most works remaining with the part.

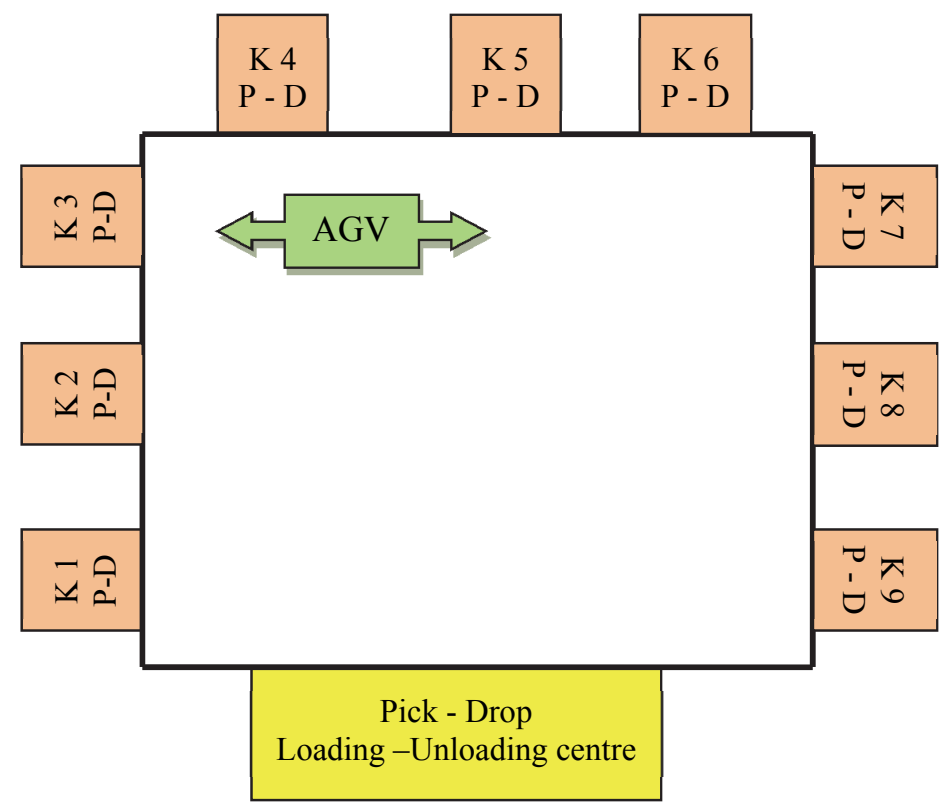

Fig. 3. The FMS configuration

Table 4

Optimum production plan and makespan according to PDRs and PHDRs

\begin{tabular}{|c|c|c|c|c|c|c|c|c|c|c|}
\hline \multirow{3}{*}{ Part routes } & \multicolumn{10}{|c|}{ Makespan } \\
\hline & \multicolumn{6}{|c|}{ Literature, Haq et al. (2003) } & \multicolumn{4}{|c|}{ Proposed PHDRs } \\
\hline & SPT & LPT & LOR & MOR & LWR & MWR & $\begin{array}{l}\text { SPS }+ \\
\text { ECT }\end{array}$ & $\begin{array}{l}\text { SWT } \\
+ \\
\text { SPS }\end{array}$ & $\begin{array}{l}\text { LWT + } \\
\text { LPS }\end{array}$ & $\begin{array}{l}\text { SPT }+ \\
\text { SPS }\end{array}$ \\
\hline $\begin{array}{c}\mathrm{E}_{1}: \mathrm{K} 2-\mathrm{K} 5-\mathrm{K} 3-\mathrm{K} 1-\mathrm{K} 8-\mathrm{K} 4-\mathrm{K} 6- \\
\mathrm{K} 9-\mathrm{K} 7 \\
\mathrm{E}_{2}: \mathrm{K} 6-\mathrm{K} 2-\mathrm{K} 1-\mathrm{K} 7-\mathrm{K} 8-\mathrm{K} 3-\mathrm{K} 4- \\
\text { K9-K5 }\end{array}$ & 549 & 626 & 676 & 626 & 659 & 549 & 548 & 611 & 619 & 562 \\
\hline $\begin{array}{c}\mathrm{E}_{1}: \mathrm{K} 2-\mathrm{K} 5-\mathrm{K} 3-\mathrm{K} 1-\mathrm{K} 8-\mathrm{K} 4-\mathrm{K} 6- \\
\mathrm{K} 9-\mathrm{K} 7 \\
\mathrm{E}_{2}: \mathrm{K} 6-\mathrm{K} 9-\mathrm{K} 5-\mathrm{K} 3-\mathrm{K} 1-\mathrm{K} 4-\mathrm{K} 7- \\
\mathrm{K} 8-\mathrm{K} 2\end{array}$ & 602 & 549 & 549 & 549 & 602 & 549 & 542 & 571 & 586 & 543 \\
\hline $\begin{array}{c}\mathrm{E}_{1}: \mathrm{K} 2-\mathrm{K} 6-\mathrm{K} 3-\mathrm{K} 5-\mathrm{K} 1-\mathrm{K} 4-\mathrm{K} 9- \\
\mathrm{K} 7-\mathrm{K} 8 \\
\mathrm{E}_{2}: \mathrm{K} 6-\mathrm{K} 2-\mathrm{K} 1-\mathrm{K} 7-\mathrm{K} 8-\mathrm{K} 3-\mathrm{K} 4- \\
\mathrm{K} 9-\mathrm{K} 5\end{array}$ & 647 & 635 & 549 & 600 & 777 & 549 & 576 & 595 & 636 & 534 \\
\hline $\begin{array}{c}\mathrm{E}_{1}: \mathrm{K} 2-\mathrm{K} 6-\mathrm{K} 3-\mathrm{K} 5-\mathrm{K} 1-\mathrm{K} 4-\mathrm{K} 9- \\
\mathrm{K} 7-\mathrm{K} 8\end{array}$ & 654 & 660 & 548 & 599 & 654 & 548 & 543 & 604 & 576 & 547 \\
\hline $\begin{array}{c}\mathrm{E}_{2}: \mathrm{K} 6-\mathrm{K} 9-\mathrm{K} 5-\mathrm{K} 3-\mathrm{K} 1-\mathrm{K} 4-\mathrm{K} 7- \\
\mathrm{K} 8-\mathrm{K} 2\end{array}$ & & & & & & & & & & \\
\hline
\end{tabular}


The part production route with least makespan is considered from the yield of Giffler and Thomson algorithm (Giffler \& Thompson, 1960). The part process route with a yield of least makespan by the application of the four priority hybrid dispatching rules and six priority dispatching rules from the literature Haq et al. (2003) is shown in the second option of table 4. The LPT, LOR, MOR, MWR, SPS+ECT, $\mathrm{SPT}+\mathrm{SPS}$ dispatching rules yield the least makespan for the parts in the FMS. The part route with the lowest makespan is considered as an input for the multiobjective sustainable scheduling between the AGVs and production centers installed in $U$ type FMS layout. So the input part route for part $E_{1}$ and $E_{2}$ is given as follows.

\section{Part E1: K2-K5-K3-K1-K8-K4-K6-K9-K7 \\ Part $\mathrm{E}_{2}$ : K6-K9-K5-K3-K1-K4-K7-K8-K2}

The distance between the production centers is mentioned in Table 5. The load-unload distance matrix between production machines is presented in table 6 , the part-production center incidence matrix for the part routes with least yield of makespan for the production schedule is shown in Table 7.

Table 5

Distance matrix between production centers

\begin{tabular}{cccccccccc}
\hline Production centre & K1 & K2 & K3 & K4 & K5 & K6 & K7 & K8 & K9 \\
\hline K1 & 0 & 6 & 8 & 12 & 14 & 14 & 12 & 8 & 6 \\
K2 & 6 & 0 & 6 & 10 & 12 & 14 & 12 & 10 & 8 \\
K3 & 8 & 6 & 0 & 8 & 10 & 12 & 10 & 9 & 7 \\
K4 & 12 & 10 & 8 & 0 & 8 & 11 & 12 & 9 & 10 \\
K5 & 14 & 12 & 10 & 8 & 0 & 7 & 9 & 12 & 6 \\
K6 & 14 & 14 & 12 & 11 & 7 & 0 & 12 & 14 & 12 \\
K7 & 12 & 12 & 10 & 12 & 9 & 12 & 0 & 4 & 6 \\
K8 & 8 & 10 & 9 & 9 & 12 & 14 & 4 & 0 & 4 \\
K9 & 6 & 8 & 7 & 10 & 6 & 12 & 6 & 4 & 0 \\
\hline
\end{tabular}

Table 6

Loading - Unloading distance matrix between the production centers

\begin{tabular}{cccccccccc}
\hline Production centre & K1 & K2 & K3 & K4 & K5 & K6 & K7 & K8 & K9 \\
\hline Loading & 2 & 4 & 6 & 7 & 12 & 12 & 12 & 11 & 14 \\
Unloading & 3 & 4 & 7 & 8 & 13 & 14 & 10 & 15 & 16 \\
\hline
\end{tabular}

Table 7

Part - Production center incidence matrix (best route considered with least makespan)

\begin{tabular}{cccccccccc}
\hline \multirow{2}{*}{ Part types } & \multicolumn{8}{c}{ Part production sequence } \\
\cline { 2 - 11 } & 1 & 2 & 3 & 4 & 5 & 6 & 7 & 8 & 9 \\
\hline $\mathrm{E}_{1}$ & $\mathrm{~K} 2$ & $\mathrm{~K} 5$ & $\mathrm{~K} 3$ & $\mathrm{~K} 1$ & $\mathrm{~K} 8$ & $\mathrm{~K} 4$ & $\mathrm{~K} 6$ & $\mathrm{~K} 9$ & $\mathrm{~K} 7$ \\
$\mathrm{E}_{2}$ & $\mathrm{~K} 6$ & $\mathrm{~K} 9$ & $\mathrm{~K} 5$ & $\mathrm{~K} 3$ & $\mathrm{~K} 1$ & $\mathrm{~K} 4$ & $\mathrm{~K} 7$ & $\mathrm{~K} 8$ & $\mathrm{~K} 2$ \\
\hline
\end{tabular}

\section{Results \& Discussion}

The resulting yield from applied grey wolf optimization algorithm mainly depends on iteration run, size of the grey wolves and parameter tuning of the GWO algorithm. In this study the GWO algorithm was run on a computer with Intel(R) Core(TM) i5 processor specifications and simulated for 300 iterations thereafter cooled off, the population size of the grey wolves in the algorithm was considered to be 50 . The parameter tuning of the grey wolf algorithm is done with two random vectors $\overrightarrow{\mathrm{r}}_{1}$ and $\overrightarrow{\mathrm{r}}_{2}$ are considered in the range of $(0,1)$ and the controlling parameter $\vec{a}$ considered to have linearly decreasing values from 
2 to 0 during the iterations. The resulting yield of GWO algorithm is compared with the resulting yield of other algorithms namely SAA and GA, from the literature Haq et al. (2003) and also presented in Table 8. The simulation yield comparison of GWO, SA, and GA from the literature Haq et al. (2003) at an iteration step interval of 10 for the multi-objective simultaneous scheduling is discussed in table 9 .

Table 8

Comparison of objective functions yields from the proposed algorithm and algorithm from literature

\begin{tabular}{cccc}
\hline Parameters & \multicolumn{2}{c}{ Literature, Haq et al. (2003) } & Proposed algorithm \\
& GA & SAA & GWO \\
\hline$Z^{*}$ & 96.99 & 103 & 134 \\
D $^{*}$ & 147 & 157 & 3 \\
B $^{*}$ & 3 & 3 & 162347598 \\
\hline O.S. & 163425798 & 142376859 & Operating Sequence O.S.
\end{tabular}

Table 9

Comparison of numerical results of the proposed evolutionary algorithms

Literature, Haq et al. (2003)

Proposed evolutionary algorithm

\begin{tabular}{|c|c|c|c|c|c|c|c|c|c|}
\hline \multirow{2}{*}{ Iterations } & \multicolumn{3}{|c|}{ GA } & \multicolumn{3}{|c|}{ SAA } & \multicolumn{3}{|c|}{ GWO } \\
\hline & $\mathrm{D}^{*}$ & $\mathrm{~B}^{*}$ & $Z^{*}$ & $\mathrm{D}^{*}$ & $\mathrm{~B}^{*}$ & $Z^{*}$ & $\mathrm{D}^{*}$ & $\mathrm{~B}^{*}$ & $Z^{*}$ \\
\hline 1 & 176 & 8 & 137.6 & 186 & 7 & 137.59 & 174 & 7 & 136.67 \\
\hline 10 & 183 & 6 & 133.8 & 174 & 9 & 135.59 & 170 & 6 & 133.34 \\
\hline 20 & 172 & 6 & 127.2 & 172 & 9 & 132 & 165 & 6 & 125.27 \\
\hline 30 & 182 & 5 & 126.8 & 189 & 5 & 130.59 & 169 & 6 & 124.93 \\
\hline 40 & 175 & 6 & 121.4 & 180 & 6 & 129.99 & 171 & 5 & 120.21 \\
\hline 50 & 164 & 7 & 120 & 175 & 7 & 128.59 & 160 & 5 & 117.76 \\
\hline 60 & 170 & 6 & 118.9 & 183 & 5 & 126.99 & 164 & 5 & 115.12 \\
\hline 70 & 159 & 6 & 117 & 162 & 8 & 126.39 & 153 & 5 & 112.32 \\
\hline 80 & 168 & 4 & 114.8 & 168 & 7 & 124 & 164 & 5 & 107.37 \\
\hline 90 & 158 & 5 & 112.8 & 153 & 9 & 121.79 & 152 & 5 & 104.47 \\
\hline 100 & 156 & 5 & 110.4 & 172 & 5 & 119.99 & 150 & 4 & 101.34 \\
\hline 110 & 165 & 3 & 109.8 & 163 & 6 & 118.19 & 142 & 4 & 98.23 \\
\hline 120 & 161 & 4 & 108.6 & 151 & 8 & 116.59 & 138 & 4 & 96.11 \\
\hline 130 & 156 & 4 & 106.4 & 161 & 5 & 113.39 & 134 & 4 & 94.15 \\
\hline 140 & 148 & 5 & 105.6 & 164 & 4 & 110.39 & 134 & 4 & 94.02 \\
\hline 150 & 147 & 3 & 96.99 & 150 & 5 & 104.39 & 134 & 3 & 93.45 \\
\hline 160 & 147 & 3 & 96.99 & 150 & 4 & 104.1 & 134 & 4 & 93.45 \\
\hline 170 & 147 & 3 & 96.99 & 157 & 3 & 103 & 134 & 3 & 93.45 \\
\hline 180 & 147 & 3 & 96.99 & 157 & 3 & 103 & 134 & 3 & 93.45 \\
\hline 190 & 147 & 3 & 96.99 & 157 & 3 & 103 & 134 & 3 & 93.45 \\
\hline 200 & 147 & 3 & 96.99 & 157 & 3 & 103 & 134 & 3 & 93.45 \\
\hline 210 & 147 & 3 & 96.99 & 157 & 3 & 103 & 134 & 3 & 93.45 \\
\hline 220 & 147 & 3 & 96.99 & 157 & 3 & 103 & 134 & 3 & 93.45 \\
\hline 230 & 147 & 3 & 96.99 & 157 & 3 & 103 & 134 & 3 & 93.45 \\
\hline 240 & 147 & 3 & 96.99 & 157 & 3 & 103 & 134 & 3 & 93.45 \\
\hline 250 & 147 & 3 & 96.99 & 157 & 3 & 103 & 134 & 3 & 93.45 \\
\hline 260 & 147 & 3 & 96.99 & 157 & 3 & 103 & 134 & 3 & 93.45 \\
\hline 270 & 147 & 3 & 96.99 & 157 & 3 & 103 & 134 & 3 & 93.45 \\
\hline 280 & 147 & 3 & 96.99 & 157 & 3 & 103 & 134 & 3 & 93.45 \\
\hline 290 & 147 & 3 & 96.99 & 157 & 3 & 103 & 134 & 3 & 93.45 \\
\hline 300 & 147 & 3 & 96.99 & 157 & 3 & 103 & 134 & 3 & 93.45 \\
\hline
\end{tabular}

$\mathrm{D}^{*}=$ Distance

$\mathrm{B}^{*}=$ No. of Backtracking

$\mathrm{Z}^{*}=$ Objective function 


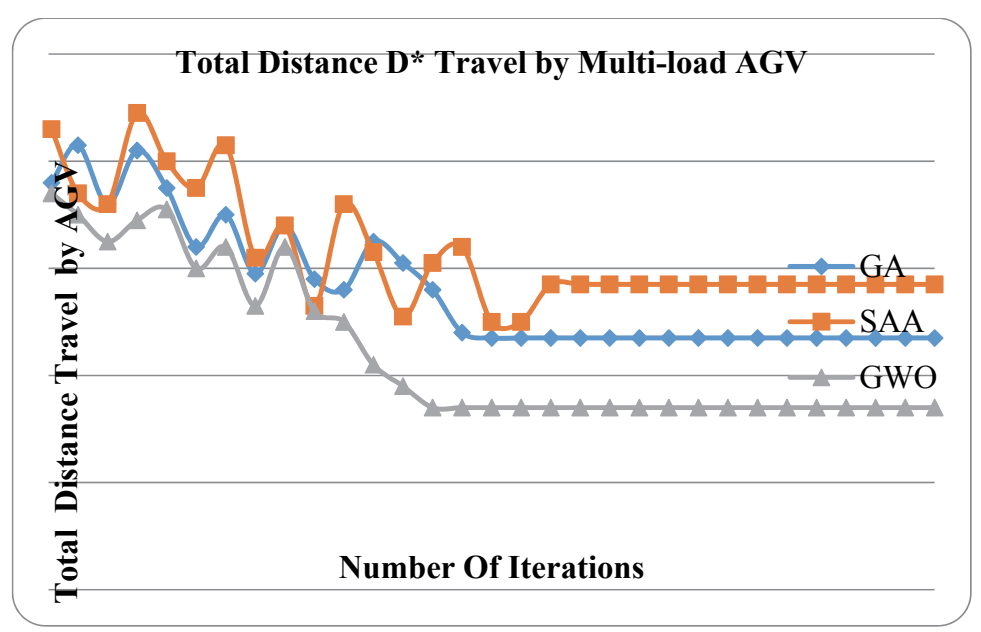

Fig. 4. Total distance travel (D*) by AGV

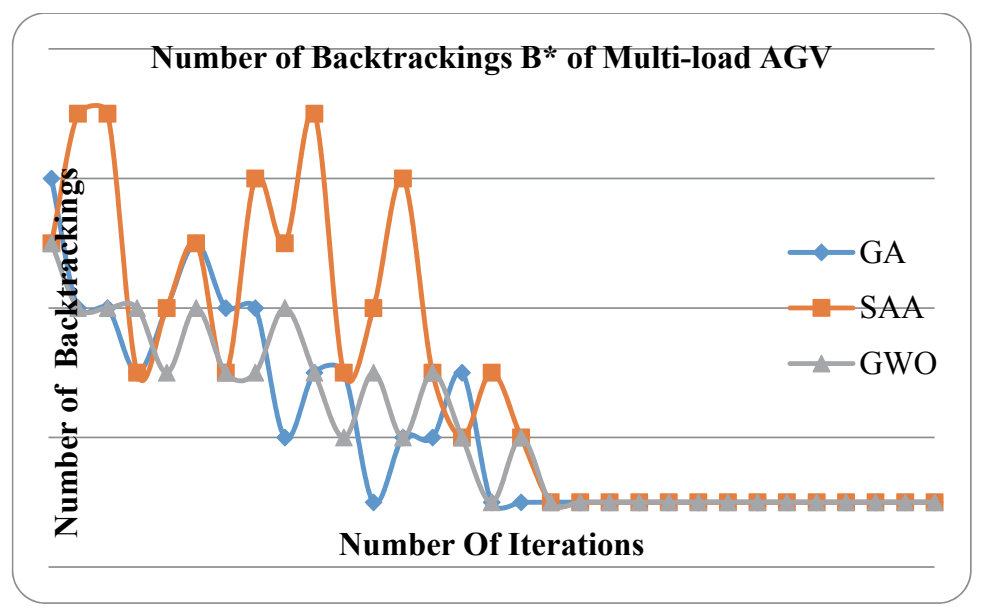

Fig. 5. Number of backtracking (B*) by AGV

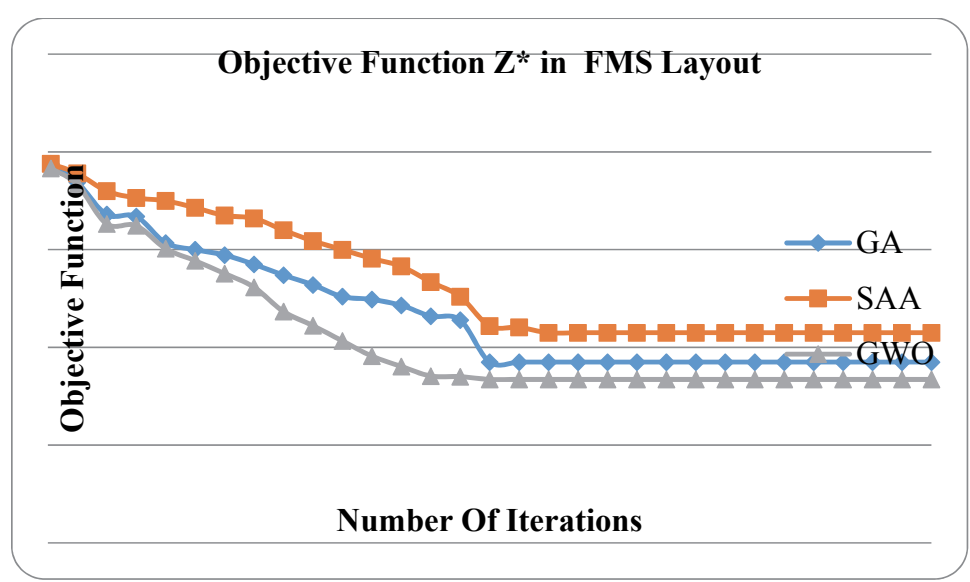

Fig. 6. Objective function (Z*) by AGV

\section{Conclusion and future work}

This paper investigates solutions for the sustainable multi-objective scheduling problems between the AGVs and FMS. In order to yield the least makespan in the production of parts, the production schedule 
is developed from the Giffler and Thomson algorithm by using priority hybrid dispatching rules. Thereafter to minimize the back-tracking and the distance travel of AGV simultaneously, the multi-objective scheduling of AGV and U type FMS layout was carried out by the grey wolf optimization (GWO) algorithm. The output yield of GWO algorithm is compared with the output yield of genetic algorithm (GA) and simulated annealing algorithm (SAA) applied on a benchmark problem from the literature Haq et al. (2003). After completion of the simulation run, the GWO algorithm found to reduce the distance travel $(D)$ of AGV by $8.84 \%$ and $14.64 \%$ in comparison to SAA and GA respectively and the GWO algorithm also reduce the overall objective function $(Z)$ to $9.55 \%$ and $3.54 \%$ in comparison to the SAA and GA respectively. However, no effect is observed in the reduction of back-tracking $(B)$ for the moving AGV in the FMS facility. The output clearly shows that the GWO can be effectively applied for the sustainable multi-objective simultaneous scheduling decisions within the FMS resources.

The research study for future can be carried out to find effective multi-objective scheduling solutions under different hybrid dispatching rules between the multi-load AGVs and other FMS resources. Various other performance parameters can also be included for investigations such as different sizes of FMS configurations, the reliability of production centers and AGVs to name a few. Further, other natureinspired optimization algorithms such as modified memetic particle swarm optimization (MMPSO) algorithm, clonal selection algorithm (CSA) may also be attempted for the solution of sustainable multiobjective simultaneous scheduling issues of various FMS resources.

\section{References}

Abdelmaguid, T. F., Nassef, A. O., Kamal, B. A., \& Hassan, M. F. (2004). A hybrid GA/heuristic approach to the simultaneous scheduling of machines and automated guided vehicles. International Journal of Production Research, 42(2), 267-281.

Angra, S., Chanda, A., \& Chawla, V. (2018). Comparison and evaluation of job selection dispatching rules for integrated scheduling of multi-load automatic guided vehicles serving in variable sized flexible manufacturing system layouts: A simulation study. Management Science Letters, 8(4), 187-200.

Bozorg-Haddad, O. (2017). Advanced Optimization by Nature-Inspired Algorithms.

Chawla, V., Chanda, A., Angra, S., \& Chawla, G. (2018a). The sustainable project management: A review and future possibilities. Journal of Project Management, 3(3), 157-170.

Chawla, V.K., Chanda, A., \& Angra, S. (2018b). A Clonal Selection Algorithm for Minimizing Distance Travel \& Back Tracking of Automatic Guided Vehicles in Flexible Manufacturing System. Journal of The Institution of Engineers (India): Series C, DOI: 10.1007/s40032-018-0447-5.

Chawla, V.K., Chanda, A., \& Angra, S. (2018c). Scheduling of multi-load AGVs in FMS by modified memetic particle swarm optimization algorithm. Journal of Project Management, 3(1), 39-54.

Chawla, V.K., Chanda, A., \& Angra, S. (2018d). Automatic guided vehicles fleet size optimization for flexible manufacturing system by grey wolf optimization algorithm. Management Science Letters, $8(2), 79-90$.

Deb, K. (2001). Multi-objective optimization using evolutionary algorithms (Vol. 16). John Wiley \& Sons.

Egbelu, P. J., \& Tanchoco, J. M. A. (1986). Potentials for bi-directional guide-path for automated guided vehicle-based systems. International Journal of Production Research, 24(5), 1075-1097.

Gaskins, R. J., \& Tanchoco, J. M. (1987). Flow path design for automated guided vehicle systems. International Journal of Production Research, 25(5), 667-676.

Giffler, B., \& Thompson, G. L. (1960). Algorithms for solving production-scheduling problems. Operations research, 8(4), 487-503.

Gnanavelbabu, A., Jerald, J., Noorul Haq, A., \& Asokan, P. (2009). Multi-objective scheduling of jobs, AGVs and AS/RS in FMS using artificial immune system. In Proceedings of National Conference on Emerging trends in Engineering and Sciences (pp. 229-239).

Ho, Y. C., \& Liu, H. C. (2009). The performance of load-selection rules and pickup-dispatching rules for multiple-load AGVs. Journal of Manufacturing Systems, 28(1), 1-10. 
Haq, A. N., Karthikeyan, T., \& Dinesh, M. (2003). Scheduling decisions in FMS using a heuristic approach. The International Journal of Advanced Manufacturing Technology, 22(5-6), 374-379.

Huang, K. L., \& Liao, C. J. (2008). Ant colony optimization combined with taboo search for the job shop scheduling problem. Computers \& Operations Research, 35(4), 1030-1046.

Jahromi, M. H. M. A., Tavakkoli-Moghaddam, R., Makui, A., \& Saghaei, A. (2017). A novel mathematical model for a scheduling problem of dynamic machine-tool selection and operation allocation in a flexible manufacturing system: A modified evolutionary algorithm. Scientia Iranica. Transaction E, Industrial Engineering, 24(2), 765.

Jain, V., \& Raj, T. (2013). Evaluation of flexibility in FMS using SAW and WPM. Decision Science Letters, 2(4), 223-230.

Jerald, J., Asokan, P., Prabaharan, G., \& Saravanan, R. (2005). Scheduling optimisation of flexible manufacturing systems using particle swarm optimisation algorithm. The International Journal of Advanced Manufacturing Technology, 25(9), 964-971.

Kaban, A. K., Othman, Z., \& Rohmah, D. S. (2012). Comparison of dispatching rules in job-shop scheduling problem using simulation: a case study. International Journal of Simulation Modelling, 11(3), $129-140$

Kashan, A. H., \& Karimi, B. (2009). A discrete particle swarm optimization algorithm for scheduling parallel machines. Computers \& Industrial Engineering, 56(1), 216-223.

Kim, S. H., \& Hwang, H. (1999). An adaptive dispatching algorithm for automated guided vehicles based on an evolutionary process. International Journal of Production Economics, 60, 465-472.

Lee, D. Y., \& Di Cesare, F. (1994). Integrated scheduling of flexible manufacturing systems employing automated guided vehicles. IEEE Transactions on Industrial Electronics, 41(6), 602-610.

Mirjalili, S., Mirjalili, S. M., \& Lewis, A. (2014). Grey wolf optimizer. Advances in Engineering Software, 69, 46-61.

Mirjalili, S., Saremi, S., Mirjalili, S. M., \& Coelho, L. D. S. (2016). Multi-objective grey wolf optimizer: a novel algorithm for multi-criterion optimization. Expert Systems with Applications, 47, 106-119.

Moghaddam, B. F., Ruiz, R., \& Sadjadi, S. J. (2012). Vehicle routing problem with uncertain demands: An advanced particle swarm algorithm. Computers \& Industrial Engineering, 62(1), 306-317.

Prakash, A., Chan, F. T., \& Deshmukh, S. G. (2011). FMS scheduling with knowledge based genetic algorithm approach. Expert Systems with Applications, 38(4), 3161-3171.

Rajotia, S., Shanker, K., \& Batra, J. L. (1998). A semi-dynamic time window constrained routeing strategy in an AGV system. International Journal of Production Research, 36(1), 35-50.

Sabuncuoglu, I., \& Hommertzheim, D. L. (1992). Dynamic dispatching algorithm for scheduling machines and automated guided vehicles in a flexible manufacturing system. The International Journal of Production Research, 30(5), 1059-1079.

Sadjadi, S. J., \& Makui, A. (2002). An algorithm to compute the complexity of a static production planning. International Journal of Engineering-Transactions A: Basics, 16(1), 57.

Sadrabadi, M. R., \& Sadjadi, S. J. (2009). A new approach to solve multiple objective programming problems. International Journal of Industrial Engineering \& Production Research, 20(1), 41-51.

Sen, K., Ghosh, S., \& Sarkar, B. (2017). Comparison of Customer Preference for Bulk Material Handling Equipment through Fuzzy-AHP Approach. Journal of The Institution of Engineers (India): Series C, 98(3), 367-377

Singh, R., \& Khan, B. (2016). Meta-hierarchical-heuristic-mathematical-model of loading problems in flexible manufacturing system for development of an intelligent approach. International Journal of Industrial Engineering Computations, 7(2), 177-190.

Suleyman, K., \& Ihsan, S. (1993). Beam search based algorithm for scheduling machines and AGVs in an FMS. In Proceedings of the Industrial Engineering Research Conference (pp. 308-312). Publ by IIE, Norcross, GA, United States

Taghaboni-Dutta, F., \& Tanchoco, J. M. A. (1995). Comparison of dynamic routeing techniques for automated guided vehicle system. International Journal of Production Research, 33(10), 2653-2669.

Tiwari, M., \& Harding, J. A. (2011). Evolutionary computing in advanced manufacturing (Vol. 73). John Wiley \& Sons. 
Udhayakumar, P., \& Kumanan, S. (2012). Integrated scheduling of flexible manufacturing system using evolutionary algorithms. The International Journal of Advanced Manufacturing Technology, 61(5), 621-635.

Ulusoy, G., \& Bilge, Ü. (1993). Simultaneous scheduling of machines and automated guided vehicles. The International Journal of Production Research, 31(12), 2857-2873.

Wang, Y. C., Chen, T., Chiang, H., \& Pan, H. C. (2016). A simulation analysis of part launching and order collection decisions for a flexible manufacturing system. Simulation Modeling Practice and Theory, 69, 80-91.

Xia, W. J., \& Wu, Z. M. (2006). A hybrid particle swarm optimization approach for the job-shop scheduling problem. The International Journal of Advanced Manufacturing Technology, 29(3), 360-366.

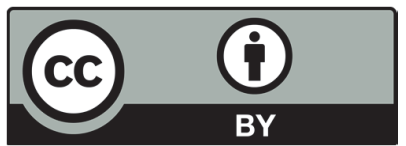

(C) 2018 by the authors; licensee Growing Science, Canada. This is an open access article distributed under the terms and conditions of the Creative Commons Attribution (CCBY) license (http://creativecommons.org/licenses/by/4.0/). 